\title{
Interações medicamentosas potenciais em adultos e idosos na atenção primária
}

\author{
Potential drug interactions in adults and the elderly in primary \\ health care
}

Janaina da Silva Santos (https://orcid.org/0000-0003-3387-7450) ${ }^{1}$

Fabiola Giordani (http://orcid.org/0000-0003-2919-856X) ${ }^{2}$

Maria Luiza Garcia Rosa (http://orcid.org/0000-0002-4508-256X) ${ }^{2}$

${ }^{1}$ Programa de PósGraduação em Saúde Coletiva, Universidade Federal Fluminense. R. Marquês do Paraná 303, Centro. 24030-210

Niterói RJ Brasil. janainasilva7@yahoo.com.br ${ }^{2}$ Departamento de Epidemiologia e Bioestatística, Universidade Federal Fluminense. Niterói RJ Brasil.

\begin{abstract}
The objective of the present study was to characterize the potential drug interactions (PDI), estimating the factors associated to their occurrence in adults and the elderly assisted by the Programa Médico de Familia de Niterói, Rio de Janeiro. This is a sub-study of STUDY DIGITALIS, which included individuals enrolled in the Niteroi Family Medical Program (45-99 years). In this study, 341 individuals with a prescription of two or more drugs were analyzed. The classification of PDI followed MICROMEDEX. There were 113 different interactions. Of the total number of individuals, $63.6 \%$ had at least one PDI. The variables with higher probability of PDI were: lower level of schooling; income less than $R \$ 800.00$; absence of health plan; previous diagnosis of hypertension and diabetes; use of 5 or more medications prescribed. After adjustment, the variables previous diagnosis of hypertension and diabetes and use of 5 or more prescription drugs remained statistically significant. Careful management of primary care treatment with monitoring is important in patients with PDI, especially in patients who are more susceptible.
\end{abstract}

Key words Drug Interactions, Primary Health Care, Outpatients.
Resumo O objetivo do presente estudo foi caracterizar as interações medicamentosas potenciais (IMP) e avaliar os fatores associados à sua ocorrência em adultos e idosos assistidos pelo Programa Médico de Família de Niterói, Rio de Janeiro. Trata-se de um subestudo do ESTUDO DIGITALIS, que incluiu indivíduos cadastrados no Programa Médico de Família de Niterói (45-99 anos). Foram analisados 341 indivíduos com prescrição de dois ou mais medicamentos. A classificação das IMP seguiu o MICROMEDEX ${ }^{\circledR}$. Houve 113 interações diferentes. Do total de indivíduos, 63,6\% apresentaram pelo menos uma IMP. As variáveis com maior chance de IMP foram: menor escolaridade; renda inferior a $R \$ 800,00$; ausência de plano de saúde; diagnóstico de hipertensão, diabetes, infarto agudo do miocárdio; e uso de 5 ou mais medicamentos prescritos. Após ajuste, as variáveis diagnóstico anterior de hipertensão $e$ diabetes e uso de 5 ou mais medicamentos prescritos permaneceram significativas estatisticamente. É importante uma gestão cautelosa do tratamento na atenção básica com monitoramento das IMP, especialmente em pacientes mais susceptíveis.

Palavras-chave Interações de Medicamentos, Atenção Primária à Saúde, Pacientes Ambulatoriais. 


\section{Introdução}

O envelhecimento populacional e a adoção por grande parte da população de um estilo de vida mais sedentário, com hábitos alimentares com excesso de sódio, açúcares e gordura, contribuem para um aumento das doenças crônicas como, hipertensão, diabetes, doenças cardíacas, que muitas vezes coexistem, exigindo na maioria dos casos, a prescrição de mais de um medicamento ${ }^{1}$. $\mathrm{O}$ uso concomitante de fármacos pode resultar em interações medicamentosas².

As interações medicamentosas podem ser definidas como fenômeno que ocorre quando os efeitos de um fármaco são modificados pela administração prévia ou simultânea de outro ${ }^{3}$. Entretanto, dada a dificuldade de comprovar a sua real ocorrência, os estudos comumente investigam as interações medicamentosas potenciais (IMPs), ou seja, interações que são conhecidas e documentadas na literatura com possibilidade de causar danos ao paciente. Ao identificá-las avalia-se o risco ao qual a população estudada está exposta ${ }^{4}$.

A prevalência de IMP em pacientes ambulatoriais relatada na literatura é de aproximadamente $50 \%{ }^{5}$, podendo chegar a mais de $80 \%{ }^{6}$. O número de medicamentos está associado à maior ocorrência de IMP ${ }^{4-6}$. Soma-se a esse achado o envelhecimento populacional, com o aumento do número de doenças crônicas e a necessidade de politerapia, estamos frente a um problema crescente de saúde pública.

No Brasil, em 2013, a Portaria no 529, de $1^{\circ}$ de abril de 2013, instituiu o Programa Nacional de Segurança do Paciente (PNSP) com o objetivo de "contribuir para a qualificação do cuidado em saúde em todos os estabelecimentos de saúde do território nacional" (art. $\left.2^{\circ}\right)^{7}$. Seus artigos recomendam uma série de normas que pretendem diminuir e prevenir a existência de incidentes nos serviços de saúde, que poderiam causar danos aos pacientes ${ }^{7}$. Dessa forma, aumenta a preocupação com ocorrências de interações medicamentosas.

A maioria dos estudos sobre interação medicamentosa aborda pacientes em ambiente hospitalar, com doenças ou medicamentos específicos ${ }^{8-10}$. Entretanto, os maiores usuários de medicamentos estão no nível ambulatorial, onde o medicamento e seu uso estão sob o cuidado do próprio paciente. Neste contexto, o presente artigo tem o objetivo de caracterizar as interações medicamentosas potenciais (IMP) e avaliar os fatores associados a sua ocorrência em adultos e idosos assistidos pelo Programa Médico de Família de Niterói, Rio de Janeiro.

\section{Materiais e Métodos}

O presente estudo faz parte do Estudo Digitalis, que teve o objetivo principal de estimar a prevalência da insuficiência cardíaca (IC) e seus estágios na população assistida pelo Programa Médico de Família de Niterói. Trata-se de um estudo transversal com indivíduos de ambos os sexos, com idade de 45 a 99 anos, cadastrados pelo Programa Médico de Família de Niterói, Estado do Rio de Janeiro, Brasil. A seleção da amostra incluiu 629 indivíduos selecionados aleatoriamente em dois estágios (setor do Programa Médico de Família de Niterói e pacientes). Rosa et al. ${ }^{11}$ apresentam o estudo Digitalis em detalhes.

Entre os meses de agosto de 2011 a novembro de 2012, 661 participantes responderam a um questionário com perguntas demográficas, sociais e sobre hábitos de vida. Além disso, foram coletadas amostras de sangue e de urina, realizadas consultas médicas, de enfermagem, de nutrição, eletrocardiograma, ecodoopler do coração e dados sobre uso de medicamentos. Para o presente estudo foram incluídos indivíduos do Estudo Digitális que utilizavam 2 ou mais medicamentos prescritos.

O estudo foi aprovado pelo Comitê de Ética em Pesquisa da Faculdade de Medicina da Universidade Federal Fluminense e todos os participantes assinaram o termo de consentimento livre e esclarecido.

Os critérios de inclusão do Estudo Digitalis foram: estar cadastrado no Programa Saúde de Família de Niterói RJ e idade maior ou igual a 45 anos. Foram excluídos indivíduos com condições clínicas que o impediam de comparecer a unidade de saúde para realização dos exames.

\section{O uso de medicamentos}

Todos os participantes foram alertados para trazerem as prescrições e as embalagens dos medicamentos em uso. O questionário sobre medicamentos incluiu as seguintes perguntas: nos últimos 3 meses o(a) senhor(a) tem tomado alguma medicação regularmente? Se sim: nome do medicamento, se o paciente havia trazido a receita, ou a embalagem, dose, frequência, se o paciente havia seguido a receita e se tomara o medicamento no dia anterior. 
(excelente, boa, regular e pobre). São incluídas também informações atualizadas clinicamente relevantes relacionadas à toxicologia, doenças, cuidados intensivos, e medicina alternativa. O material que o sistema oferece é revisado por especialistas, e atualizado periodicamente. Ele possui referências à literatura, primária e secundária. Inclui também links para acesso às referências dos artigos científicos ${ }^{12}$.

Os dados sobre medicamentos, no Estudo Digitalis, foram registrados no banco de dados em sua forma comercial ou pelo nome do princípio ativo. Foi calculada a frequência dos medicamentos no SPSS (versão 21) para possibilitar identificar as repetições, e assim também foi gerada uma lista única dos medicamentos que foram usados pelo menos por um paciente. Esta lista foi a base para a padronização da nomenclatura destes medicamentos, os quais, quando necessário, foram reescritos pelo princípio ativo utilizando a Denominação Comum Brasileira (DCB ${ }^{13}$.

No total foram 115 medicamentos para análise de suas interações. Os medicamentos listados pelo nome do princípio ativo foram traduzidos para o inglês, uma vez que o Micromedex ${ }^{\circledR}$ só permite a pesquisa neste idioma.

A lista de medicamentos foi introduzida no Micromedex ${ }^{\circledR}$ e as interações medicamentosas potenciais identificadas. Os pares das interações medicamentosas foram registrados e localizados no banco dos pacientes que os utilizaram concomitantemente.

As interações medicamentosas potenciais foram classificadas segundo a gravidade e grau de documentação de acordo com o Micromedex ${ }^{\circledR 12}$.

Foram criadas variáveis dos pares das interações e também para verificação de interação em relação a cada indivíduo para que fosse possível realizar as análises.

\section{Análise estatística}

As informações foram apresentadas como frequência absoluta e relativa e a diferença entre os grupos com e sem IMP foram testadas com o teste do qui quadrado de Pearson, com correções de continuidade, sempre que necessário. Para as

variáveis com nível de significância estatísticas $<0,20$ foram estimados os OR brutos e seus intervalos de confiança a 95\% por regressão logística simples. Os OR ajustados foram estimados por modelo logístico múltiplo, incluindo as variáveis com $\mathrm{p}<0,20$ nas regressões logísticas simples.

A análise dos dados foi realizada no software SPSS versão 21.0 (SPSS inc, Chicago, IL).

Foram descritas as dez interações graves e moderadas mais prevalentes.

\section{Resultados}

Dos 661 indivíduos do estudo de base, 203 não usavam medicamentos, 105 usavam 1 e 12 não havia informação sobre medicamentos. No presente estudo foram incluídos os 341 (51\%) participantes investigados pelo Estudo Digitalis, que utilizavam 2 ou mais medicamentos.

Foram prescritos 115 medicamentos distintos com 113 interações medicamentosas potenciais diferentes entre eles. $\mathrm{O}$ número de medicamentos prescritos variou de dois (critério de inclusão para o presente estudo) a 10, com média de 3,72 $(\mathrm{DP}=0,94)$.

Do total de indivíduos investigados, 217 $(63,6 \%)$ apresentaram pelo menos uma IMP, variando de 0 a 13 e média de 1,23 ( $\mathrm{DP}=0,09)$. Quarenta e quatro do total de pacientes $(12,9 \%)$ apresentaram pelo menos uma IMP grave, 205 $(60,1 \%)$ pelo menos uma moderada e $13(3,8 \%)$ somente interações leves. Os percentuais em relação ao total de interações foram respectivamente 20,3\%; 9,5\% e 6,0\% (Tabela 1). Não foram identificadas interações contraindicadas.

A Tabela 1 apresenta também a caracterização dos indivíduos incluídos. Trata-se de uma população de maioria de mulheres, com estudo até a $5^{\mathrm{a}}$ série e com renda per capita até $\mathrm{R} \$ 800,00$, sem plano de saúde e não fumante. Mais de $86,5 \%$ tinham diagnóstico anterior de hipertensão e $30,8 \%$ de diabetes.

Um percentual maior de pacientes que usavam 5 ou mais medicamentos $(82,7 \%)$ em relação àqueles que usavam até 4 medicamentos (55,3\%), número de pacientes que possuíam 3 ou mais comorbidades $(75 \%)$ em relação àqueles que possuíam até 2 comorbidades (59,9\%) e aqueles pacientes que apresentaram doenças sujeitos a interação, como a hipertensão $(69,5 \%)$, diabetes $(79 \%)$ e infarto agudo do miocárdio $(81,8 \%)$ (Tabela 2).

Os indivíduos que apresentaram maior chance de ocorrências de IMP foram aqueles com menor escolaridade, com renda até $\mathrm{R} \$ 800,00$ 
Tabela 1. Caracterização dos pacientes estudados (45 a 99 anos) por variáveis sociodemográficas e de saúde. Município Niterói em 2011-2012 (N=341).

\begin{tabular}{|c|c|c|}
\hline Variável & $\mathbf{N}$ & $\begin{array}{c}\text { Porcentagem } \\
(\%)\end{array}$ \\
\hline \multicolumn{3}{|l|}{$\begin{array}{l}\text { Interações medicamentosas } \\
\text { potenciais (IMP) }\end{array}$} \\
\hline $\begin{array}{l}\text { Pelo menos uma IMP de } \\
\text { qualquer gravidade }\end{array}$ & 217 & $63,6^{\#}$ ou $100,0^{*}$ \\
\hline Pelo menos uma IMP grave & 44 & $12,9^{*}$ ou $20,3^{*}$ \\
\hline $\begin{array}{l}\text { Pelo menos uma IMP } \\
\text { moderada }\end{array}$ & 205 & $60,1^{\#}$ ou $95,0^{*}$ \\
\hline Somente IMP leve & 13 & $3,8^{\#}$ ou $6,0^{*}$ \\
\hline \multicolumn{3}{|l|}{ Características da população } \\
\hline \multicolumn{3}{|l|}{ Sexo } \\
\hline Masculino & 104 & 30,5 \\
\hline Feminino & 237 & 69,5 \\
\hline \multicolumn{3}{|l|}{ Idade } \\
\hline $60 \mathrm{e}+$ & 177 & 51,9 \\
\hline 45-59 & 164 & 48,1 \\
\hline \multicolumn{3}{|l|}{ Cor da pele } \\
\hline Branco & 110 & 32,3 \\
\hline Pardo & 130 & 38,1 \\
\hline Preto & 97 & 28,1 \\
\hline \multicolumn{3}{|l|}{ Escolaridade } \\
\hline $5^{\mathrm{a}}$ série e + & 187 & 54,8 \\
\hline até $4^{\mathrm{a}}$ série & 153 & 44,9 \\
\hline \multicolumn{3}{|l|}{ Renda } \\
\hline$>800$ reais per capita & 82 & 24,0 \\
\hline Até 800 reais per capita & 255 & 74,8 \\
\hline \multicolumn{3}{|l|}{ Possui plano de Saúde } \\
\hline Sim & 60 & 17,6 \\
\hline Não & 278 & 81,5 \\
\hline
\end{tabular}

(salário mínimo regional na época da coleta), sem plano de saúde, com diagnóstico anterior de hipertensão, diabetes e infarto agudo do miocárdio, com 3 ou mais comorbidades e com 5 ou mais medicamentos prescritos (polifarmácia). Após ajuste, as variáveis diagnóstico anterior de hipertensão e diabetes e uso de 5 ou mais medicamentos prescritos permaneceram significativas estatisticamente (Tabela 3 ).

$\mathrm{Na}$ Tabela 4 observa-se as 10 IMP mais frequentes encontradas classificadas como de maior gravidade e as com gravidade moderada. Pode-se destacar como mais frequente a interação entre os medicamentos anlodipino e sinvastatina entre as graves e entre os medicamentos inibidores da ECA e diuréticos tiazídicos para as moderadas.
Tabela 1. Caracterização dos pacientes estudados (45 a 99 anos) por variáveis sociodemográficas e de saúde. Município Niterói em 2011-2012 (N=341).

\begin{tabular}{|c|c|c|}
\hline Variável & $\mathbf{N}$ & $\begin{array}{c}\text { Porcentagem } \\
(\%)\end{array}$ \\
\hline \multicolumn{3}{|l|}{ Fumo Atual } \\
\hline Sim & 47 & 13,8 \\
\hline Não & 294 & 86,2 \\
\hline \multicolumn{3}{|l|}{ Estado de Saúde } \\
\hline Excelente & 13 & 3,8 \\
\hline Muito bom & 33 & 9,7 \\
\hline Bom & 209 & 61,3 \\
\hline Ruim & 66 & 19,4 \\
\hline Muito ruim & 18 & 5,3 \\
\hline \multicolumn{3}{|l|}{ Comorbidades } \\
\hline Asma/Bronquite & 50 & 14,7 \\
\hline Hipertensão & 295 & 86,5 \\
\hline Diabetes & 105 & 30,8 \\
\hline Obesidade & 66 & 19,4 \\
\hline Doença Renal & 28 & 8,2 \\
\hline Depressão & 66 & 19,4 \\
\hline $\begin{array}{l}\text { Infarto agudo do } \\
\text { miocárdio }\end{array}$ & 22 & 6,5 \\
\hline \multicolumn{3}{|l|}{ Número de comorbidades } \\
\hline Até 2 & 257 & 75,4 \\
\hline 3 ou mais & 84 & 24,6 \\
\hline \multicolumn{3}{|l|}{$\begin{array}{l}\text { Internação nos últimos } 12 \\
\text { meses }\end{array}$} \\
\hline Sim & 27 & 7,9 \\
\hline Não & 312 & 91,5 \\
\hline \multicolumn{3}{|l|}{ Polifarmácia } \\
\hline Até 4 medicamentos & 237 & 69,5 \\
\hline 5 ou mais medicamentos & 104 & 30,5 \\
\hline
\end{tabular}

\section{Discussão}

A prevalência de IMP foi de $63,6 \%$, considerando somente os indivíduos com prescrição de pelo menos dois medicamentos, sendo de $32,8 \%$ se considerarmos todos os indivíduos investigados pelo Estudo Digitalis.

Este percentual é comparável ao estudo de Mibielli e colaboradores ${ }^{4}$ que incluiu 261 usuários de anti-hipertensivos aposentados e pensionistas com idade superior a 60 anos. O estudo de Bleich e colaboradores ${ }^{14}$ apresenta população com características demográficas e sociais semelhantes à população aqui avaliada, incluiu 96 indivíduos em um inquérito populacional e encontraram $66,7 \%$ de IMP. Doubova et al. ${ }^{15}$ 
Tabela 2. Caracterização dos pacientes estudados ( 45 a 99 anos) sujeitos a interação medicamentosa potencial (IMP) ou não, por variáveis sociodemográficas e de saúde. Município de Niterói em 2011-2012 (N=341).

\begin{tabular}{|c|c|c|c|}
\hline Variável & Sem IMP (\%) & Com IMP (\%) & P-valor ${ }^{\star}$ \\
\hline \multicolumn{4}{|l|}{ Sexo } \\
\hline Masculino & $35(33,7 \%)$ & $69(66,3 \%)$ & 0,491 \\
\hline Feminino & $89(37,6 \%)$ & $148(62,4 \%)$ & \\
\hline Idade & & & 0,227 \\
\hline $60 \mathrm{e}+$ & $59(33,3 \%)$ & $118(66,7 \%)$ & \\
\hline $45-59$ & $65(39,6 \%)$ & $99(60,4 \%)$ & \\
\hline Cor da pele & & & 0,397 \\
\hline Branco & $45(40,9 \%)$ & $65(61,8 \%)$ & \\
\hline Pardo & $46(35,4 \%)$ & $84(65,4 \%)$ & \\
\hline Preto & $31(32,0 \%)$ & $66(70,1 \%)$ & \\
\hline \multicolumn{4}{|l|}{ Escolaridade } \\
\hline $5^{\mathrm{a}}$ série e + & $76(40,6 \%)$ & $111(59,4 \%)$ & 0,077 \\
\hline até $4^{\text {a }}$ série & $48(31,4 \%)$ & $105(68,6 \%)$ & \\
\hline Renda & & & 0,072 \\
\hline Acima de 800 reais per capita & $37(45,1 \%)$ & $45(54,9 \%)$ & \\
\hline Até 800 reais per capita & $87(34,1 \%)$ & $168(65,9 \%)$ & \\
\hline Possui plano de Saúde & & & 0,141 \\
\hline Sim & $27(45,0 \%)$ & $33(55,0 \%)$ & \\
\hline Não & $97(34,9 \%)$ & $181(65,1 \%)$ & \\
\hline Fumo Atual & & & 0,533 \\
\hline Sim & $19(40,4 \%)$ & $28(59,6 \%)$ & \\
\hline Não & $105(35,7 \%)$ & $189(64,3 \%)$ & \\
\hline Estado de Saúde & & & 0,318 \\
\hline Excelente & $3(23,1)$ & $10(76,9 \%)$ & \\
\hline Muito bom & $9(27,3 \%)$ & $24(72,7 \%)$ & \\
\hline Bom & $82(39,2 \%)$ & $127(60,8 \%)$ & \\
\hline Ruim & $26(39,4 \%)$ & $40(60,6 \%)$ & \\
\hline Muito ruim & $4(22,2 \%)$ & $14(77,8 \%)$ & \\
\hline \multicolumn{4}{|l|}{ Comorbidades } \\
\hline Asma/Bronquite & $19(38 \%)$ & $31(62 \%)$ & 0,795 \\
\hline Hipertensão & $90(30,5 \%)$ & $205(69,5 \%)$ & 0,000 \\
\hline Diabetes & $22(21 \%)$ & $83(79 \%)$ & 0,000 \\
\hline Obesidade & $24(36,4 \%)$ & $42(63,6 \%)$ & 1,00 \\
\hline Doença Renal & $10(35,7 \%)$ & $18(64,3 \%)$ & 0,941 \\
\hline Depressão & $25(37,9 \%)$ & $41(62,1 \%)$ & 0,776 \\
\hline Infarto agudo do miocárdio & $4(18,2 \%)$ & $18(81,8 \%)$ & 0,067 \\
\hline Número de comorbidades & & & 0,013 \\
\hline Até 2 & $103(40,1 \%)$ & $154(59,9 \%)$ & \\
\hline 3 ou mais & $21(25 \%)$ & $63(75,0 \%)$ & \\
\hline Internação nos últimos 12 meses & & & 0,376 \\
\hline $\operatorname{Sim}$ & $12(44,4 \%)$ & $15(55,6 \%)$ & \\
\hline Não & $112(35,9 \%)$ & $200(64,1 \%)$ & \\
\hline Polifarmácia & & & 0,000 \\
\hline Até 4 medicamentos & $106(44,7 \%)$ & $131(55,3 \%)$ & \\
\hline 5 ou mais medicamentos & $18(17,3 \%)$ & $86(82,7 \%)$ & \\
\hline
\end{tabular}

Teste do Qui-quadrado de Pearson com correção de continuidade quando necessário.

avaliaram 624 indivíduos com idade superior a 50 anos que estavam em tratamento medicamen- toso para dor crônica, assistidos em clínicas da família da Cidade do México, onde o percentual 
de IMP foi de $80 \%$. Doubova e colaboradores ${ }^{15}$ justificam tal percentual por tratar de uma população em que aproximadamente $90 \%$ fazia uso de AINES, classe de medicamentos com alta frequência de envolvimento em IMP. No presente estudo, cerca de $10 \%$ dos indivíduos faziam uso desta classe de medicamentos. Bleich e colaboradores $^{14}$, para identificar as IMP, utilizaram além do Micromedex ${ }^{\circledR}$, as bases DrugDigest ${ }^{\circledR}$, Medsca$p e^{\circledR}$ o que pode ter aumentado a sensibilidade da identificação das IMP. É interessante notar que o percentual de hipertensos no estudo de Doubova e colaboradores ${ }^{15}$ também foi elevado $(67 \%)$ e no estudo de Bleich e colaboradores ${ }^{14}$, a maioria das prescrições envolviam o sistema cardiovascular.

Em estudos na atenção primária, as IMP de gravidade moderada são as mais frequentes ${ }^{4,14-18}$. No presente estudo, as interações moderadas estiveram presentes em $95 \%$ dos pacientes que apresentaram pelo menos uma IMP e envolveram, em maior proporção, medicamentos comumente prescritos na atenção básica utilizados para hipertensão e diabetes (inibidores da enzima conversora da angiotensina, diuréticos tiazídicos, diuréticos de alça, diuréticos poupadores de potássio e medicamentos hipoglicemiantes), doenças de maior prevalência. A maior frequência de interações moderadas envolvendo medicamentos anti-hipertensivos e hipoglicemiantes também foi encontrada em outros estudos ${ }^{19,20}$.

Combinação de diuréticos e beta bloqueadores, com ou sem os inibidores da enzima conversora de angiotensina, apesar de recomendada pelas VI Diretrizes Brasileiras de Hipertensão $0^{21}$ para otimizar o efeito anti-hipertensivo, pode causar danos e são classificadas quanto a gravidade da IMP como moderada ${ }^{12}$, justificando a importância de monitorar os possíveis efeitos da associação. Ressalta-se, entretanto, que entre as interações medicamentosas potenciais mais frequentes, o grau de evidência da maioria delas é fraco ${ }^{12}$.

A associação do medicamento anlodipino com o medicamento sinvastatina foi a IMP mais frequente de maior gravidade $(14,2 \%)$. A sinvastatina é um medicamento amplamente usado para o controle do colesterol sanguíneo, no uso concomitante com o medicamento anlodipino pode ocorrer o aumento da exposição de sinvastatina colocando os pacientes em risco aumentado de miopatia e rabidomiólise. Pode causar também danos no fígado. $\mathrm{O}$ uso da sinvastatina já exige vigilância, ainda mais quando os pacientes são idosos. Quando associado ao anlodipino, a dose recomendada de sinvastatina é no máximo de $20 \mathrm{mg}^{22}$. A interação entre o medicamento amiodarona e sinvastatina também foi identificada neste estudo com frequência de $1,8 \%$ e possui as mesmas consequências clínicas apresentadas entre os medicamentos sinvastatina e anlodipi$\mathrm{no}^{12}$.

A segunda interação potencial mais frequente entre as consideradas de gravidade maior foi entre os medicamentos digoxina e espironolactona $(4,4 \%)$. O medicamento espironolactona é um diurético poupador de potássio que pode aumentar a concentração plasmática da digoxina quando usado em associação, pois reduz a depuração renal da digoxina ${ }^{12}$. Venturini e colaboradores ${ }^{17}$ identificaram esta interação em seu estudo com uma frequência de $0,9 \%$.

Outra interação potencial identificada com gravidade maior foi do uso concomitante dos medicamentos IECA e losartana (3,5\%). Quando há a associação desses dois medicamentos, o benefício é inferior às consequências adversas que podem ocorrer como: insuficiência renal, hipercalemia e hipotensão $\mathrm{o}^{21}$. A quarta IMP grave em frequência foi entre os medicamentos atenolol e clonidina $(2,7 \%)$ que pode gerar a potencialização do efeito anti-hipertensivo aumentando o risco de bradicardia sinusal ${ }^{12}$.

A interação entre digitálico e diurético tiazídico também foi identificada no estudo $(1,8 \%)$, podendo ocorrer uma deficiência de potássio e assim ocasionar uma toxicidade digitálica. Dependendo da concentração da digoxina, a toxicidade aumenta conforme os níveis de potássio vão decaindo. Pode também ocorrer uma deficiência de magnésio, nestes casos deve haver monitoramento do potássio e magnésio quando e se necessário deve ser efetuada a reposição desses eletrólitos ${ }^{3}$.

A interação enalapril e espironolactona foi identificada na frequência de $1,8 \%$, pode ocorrer efeito hipotensor aditivo ou desencadear hipercalemia ${ }^{12}$.

Quanto as IMP moderadas, a mais frequente foi entre os medicamentos enalapril e hidroclorotiazida $(52,2 \%)$ seguida da interação entre captopril e hidroclorotiazida $(43,4 \%)$. Este tipo de associação tem vantagens clínicas que superam $\mathrm{o}$ risco do desencadeamento de hipotensão postural por vasodilatação $0^{21}$.

Foram identificadas também interações de gravidade moderada entre IECA e metformina. Em relação à interação com enalapril a frequência foi de $23,0 \%$ e em relação ao captopril foi de $17,7 \%$. Quando acontece esta interação, ocorre o aumento do efeito hipoglicemiante da metformina, ou seja, aumenta o risco de hipoglicemia ${ }^{12}$. 
As VI Diretrizes Brasileiras de Hipertensão ${ }^{21}$ chama atenção para a importância de se conhecer as principais interações de anti-hipertensivos e medicamentos de uso contínuo que podem ser prescritos para o paciente hipertenso e cita as principais interações e seus efeitos, não se referindo a estratégias alternativas ao tratamento.

Observa-se que a maioria dos medicamentos envolvidos com a IMP são usados para o tratamento de problemas de hipertensão e diabetes. O Ministério da Saúde tem diversas ações na tentativa de garantir ao diagnóstico e tratamento dessas doenças crônicas, entre elas o Sistema de Cadastramento e Acompanhamento de Hipertensos e Diabéticos (HIPERDIA) ${ }^{23}$ e o Programa Farmácia Popular ${ }^{24}$. Entretanto, a realização do diagnóstico precoce e o acesso aos medicamentos necessários ao tratamento não garantem a efetividade do controle da pressão arterial e da glicemia, o uso dos medicamentos deve ser acompanhado e monitorado para favorecer a adesão e interceptar possíveis problemas relacionados ao uso dos medicamentos.

Inúmeros estudos apontam que mulheres e indivíduos com mais idade têm maior risco de IMP $^{6,12,17}$. No entanto, há numerosos estudos em que o risco de IMP para homens e mulheres é próximo e não é maior em faixas etárias mais elevadas, como aconteceu no presente estudo ${ }^{4,19,25}$. A população assistida por programas de saúde da família, como a do presente estudo, é monitorada, há busca ativa e grande parte dos medicamentos são distribuídos gratuitamente, o que pode diminuir as diferenças culturais entre homens e mulheres. Por outro lado, no presente estudo, há prevalência de hipertensão praticamente $70 \%$, o que pode ter aproximado o percentual de IMP entre as duas faixas de idade.

A maior prevalência de IMP entre indivíduos com menor escolaridade e renda, também é um achado consistente com outros estudos em populações diferentes ${ }^{25}$. Mesmo em sociedades com acesso universal tanto aos serviços de saúde, como a medicamentos, indivíduos com menor renda utilizam menos os serviços de saúde, tem menor adesão ao tratamento, o que de forma indireta, poderia ajudar a entender o fato de pacientes com menor escolaridade e renda apresentarem maior prevalência de IMP.

A associação da IMP com a polifarmácia, ou com o maior número de medicamentos prescritos e com o número de comorbidades é um achado consistente na literatura ${ }^{4,6,19,25,26}$ e também foi observado no presente estudo.
As comorbidades associadas à IMP foram a hipertensão, o diabetes e o infarto agudo do miocárdio auto-referidos, também reportado com outras populações ${ }^{27}$. Após ajuste, apenas a hipertensão e o diabetes mantiveram a significância estatística (Tabela 3), mas a chance de IMP foi praticamente o dobro para aqueles com diagnóstico anterior de infarto, ajustadas. Como observado na Tabela 4, as IMP mais frequentes envolvem os medicamentos para o tratamento das doenças crônicas hipertensão e diabetes, o que reforça a necessidade de monitoramento e acompanhamento desse grupo de pacientes.

Este estudo foi um estudo seccional e algumas limitações que podem ter levado à subestimação do percentual de IMP devem ser ressaltadas. Os dados sobre medicamentos foram coletados a partir da pergunta "nos últimos 3 meses o(a) senhor(a) tem tomado alguma medicação regular-

Tabela 3. Fatores Associados à ocorrência de interações medicamentosas potenciais em pacientes de 45 a 99 anos. Município de Niterói em 2011-2012 (N=371)

\begin{tabular}{|c|c|c|}
\hline Variáveis & $\begin{array}{c}\text { OR bruto (IC } \\
95 \%)\end{array}$ & $\begin{array}{l}\text { OR ajustado } \\
\text { (IC95\%) }\end{array}$ \\
\hline \multicolumn{3}{|l|}{ Escolaridade } \\
\hline $5^{\text {a }}$ série e + & 1 & 1 \\
\hline até $4^{\text {a }}$ série & $1,35(0,86-2,12)^{*}$ & $1,26(0,75-2,12)$ \\
\hline \multicolumn{3}{|l|}{ Renda } \\
\hline $\begin{array}{l}\text { Acima de } 800 \\
\text { reais per capita }\end{array}$ & 1 & 1 \\
\hline $\begin{array}{l}\text { Até } 800 \text { reais per } \\
\text { capita }\end{array}$ & $1,57(0,94-2,62)^{\star}$ & $1,58(0,86-2,86)$ \\
\hline \multirow{2}{*}{\multicolumn{3}{|c|}{$\begin{array}{l}\text { Possui plano de } \\
\text { Saúde }\end{array}$}} \\
\hline & & \\
\hline Sim & 1 & 1 \\
\hline Não & $1,57(0,89-2,78)^{*}$ & $1,12(0,57-2,23)$ \\
\hline \multicolumn{3}{|l|}{ Comorbidades ${ }^{@}$} \\
\hline Asma/Bronquite & $0,92(0,50-1,71)$ & \\
\hline Hipertensão & $6,45(3,19-13,04)^{*}$ & $6,12(2,89-12,95)$ \\
\hline Diabetes & $2,87(1,68-4,91)^{\star}$ & $2,48(1,29-4,77)$ \\
\hline Obesidade & $1,00(0,57-1,75)$ & \\
\hline Doença Renal & $1,03(0,46-2,31)$ & \\
\hline Depressão & $0,92(0,53-1,61)$ & \\
\hline Infarto & $2,71(0,90-8,21)^{*}$ & $1,90(0,50-7,17)$ \\
\hline \multicolumn{3}{|l|}{ Polifarmácia } \\
\hline $\begin{array}{l}\text { Até } 4 \\
\text { medicamentos }\end{array}$ & 1 & 1 \\
\hline $\begin{array}{l}5 \text { ou mais } \\
\text { medicamentos }\end{array}$ & $3,87(2,19-6,83)^{*}$ & $2,43(1,22-4,84)$ \\
\hline
\end{tabular}


Tabela 4. As dez IMP graves e as moderadas mais frequentes. Niterói em 2011-2012.

\begin{tabular}{|c|c|c|c|}
\hline $\begin{array}{l}\text { Interação medicamentosa } \\
\text { potencial }\end{array}$ & $\mathbf{N}$ & $\begin{array}{l}\text { Grau de } \\
\text { evidência }\end{array}$ & Possível consequência clínica \\
\hline \multicolumn{4}{|l|}{ Graves } \\
\hline Anlodipino-sinvastatina & 16 & Bom & $\begin{array}{l}\text { Pode ocorrer o aumento da exposição da sinvastatina e } \\
\text { aumento no risco de miopatia e rabdomiólise }\end{array}$ \\
\hline Digoxina-espironolactona & 5 & Bom & Pode aumentar a concentração da digoxina \\
\hline Enalapril-losartana & 4 & Excelente & $\begin{array}{l}\text { Pode aumentar o risco de eventos adversos como: } \\
\text { hipotensão, síncope, hipercalemia, alterações na função } \\
\text { renal, insuficiência renal aguda }\end{array}$ \\
\hline Atenolol-clonidina & 3 & Fraco & Pode aumentar o risco de bradicardia sinusal \\
\hline Captopril-losartana & 3 & Excelente & $\begin{array}{l}\text { Pode aumentar o risco de eventos adversos como: } \\
\text { hipotensão, síncope, hipercalemia, alterações na função } \\
\text { renal, insuficiência renal aguda }\end{array}$ \\
\hline Amiodarona-sinvastatina & 2 & Excelente & $\begin{array}{l}\text { Pode ocorrer o aumento da exposição da sinvastatina e } \\
\text { aumento no risco de miopatia e rabdomiólise }\end{array}$ \\
\hline Digoxina- hidroclorotiazida & 2 & Excelente & $\begin{array}{l}\text { Pode desencadear toxidade digitálica (náusea, vômito, } \\
\text { arritmias) }\end{array}$ \\
\hline Enalapril- espironolactona & 2 & Bom & Pode desencadear hipercalemia \\
\hline Fluoxetina-ibuprofeno & 2 & Excelente & Pode aumentar o risco de hemorragia \\
\hline Amiodarona-carvedilol & 1 & Excelente & $\begin{array}{l}\text { Pode desencadear hipotensão, bradicardia ou parada } \\
\text { cardíaca }\end{array}$ \\
\hline \multicolumn{4}{|l|}{ Moderadas } \\
\hline Enalapril-hidroclorotiazida & 59 & Fraco & $\begin{array}{l}\text { Pode desencadear hipotensão postural /efeito de primeira } \\
\text { passagem }\end{array}$ \\
\hline Captopril-hidroclorotiazida & 49 & Fraco & $\begin{array}{l}\text { Pode desencadear hipotensão postural /efeito de primeira } \\
\text { passagem }\end{array}$ \\
\hline Enalapril-metformina & 26 & Fraco & Pode aumentar o risco de hipoglicemia \\
\hline Captopril-metformina & 20 & Fraco & Pode aumentar o risco de hipoglicemia \\
\hline Atenolol-metformina & 18 & Bom & Pode aumentar hipoglicemia ou hiperglicemia \\
\hline Hidroclorotiazida-propranolol & 16 & Fraco & Pode desencadear hiperglicemia, hipertrigliceremia \\
\hline Enalapril-furosemida & 8 & Bom & $\begin{array}{l}\text { Pode desencadear hipotensão postural /efeito de primeira } \\
\text { passagem }\end{array}$ \\
\hline Insulina-metformina & 7 & Fraco & Pode aumentar o risco de hipoglicemia \\
\hline Captopril-furosemida & 5 & Bom & Pode aumentar o risco de hipotensão postural \\
\hline Levotiroxina-sinvastatina & 5 & Bom & Pode resultar no decréscimo da ação da levotiroxina \\
\hline
\end{tabular}

mente?", sendo assim, se no início do tratamento uma interação medicamentosa pudesse ter levado algum dano ao paciente, esse poderia ter sido interceptado por algum profissional de saúde e o medicamento substituído pelo médico, subestimando as estimativas e por outro lado, IMP aqui identificadas podem ter sido avaliadas por profissional de saúde no início do estudo, e essa não ter levado a problemas ao paciente. Outros problemas que podem ter afetado as estimativas são a utilização apenas do Micromedex ${ }^{\circledR}$ para a identificação das interações, ter sido considerado somente pares de medicamentos e não combinações de mais medicamentos, e a perda de informação sobre medicamentos em uso pelo partici- pante não ter trazido prescrição ou embalagens, conforme solicitado.

Por outro lado, trata-se de um estudo com população assistida na atenção básica, selecionada aleatoriamente o que permite que os resultados possam ser aplicáveis a outras populações adscritas a Programas de Saúde da Família.

\section{Conclusão}

Considerando o total de indivíduos investigados pelo Estudo Digitalis, 32,8\% estavam sujeitos a pelo menos uma IMP e entre aqueles com prescrição de pelo menos duas medicações o per- 
centual chegou a $63,6 \%$, em sua maioria moderadas e graves. Observou-se que portadores de hipertensão, diabetes e aqueles com diagnóstico anterior de infarto agudo do miocárdio tiveram chance aumentada de IMP. Um grande número de associações envolvendo medicações cardiovasculares como diuréticos, betabloqueadores ou IECA, observadas foram classificadas como IMP moderadas ou graves, no entanto, seus efeitos clínicos justificam suas prescrições. Apesar da

\section{Colaboradores}

JS Santos contribuiu com a concepção do estudo, análise e interpretação dos resultados e redação do manuscrito. F Giordani contribuiu com a concepção do estudo, análise e interpretação dos resultados. MLG Rosa contribuiu com a concepção do estudo, análise e interpretação dos resultados. Todos os autores aprovaram a versão final do manuscrito.
VI Diretrizes Brasileiras de Hipertensão ${ }^{21}$ alertar para as possíveis interações, observou-se que as prescrições incluíram associações que potencialmente podem causar dano e devem ser monitoradas. Conclui-se que é importante haver uma gestão mais cautelosa do tratamento na atenção básica com monitoramento dos efeitos das associações medicamentosas usadas, especialmente em pacientes com fatores associados para IMP.

\section{Referências}

1. Ramos LR. Fatores determinantes do envelhecimento saudável em idosos residentes em centro urbano: Projeto Epidoso, São Paulo. Cad Saude Publica 2003;19(3):793-798.

2. Gallagher LP. The potential for adverse drug reactions in elderly patients. Appl Nurs Res 2001;14(4):220-224.

3. Tatro DS. Drug interaction facts. St. Louis: Wolters Kluwer Health; 2011.

4. Mibielli P, Rozenfeld S, Matos GC, Acurcio FA. Interações medicamentosas potenciais entre idosos em uso dos anti-hipertensivos da Relação Nacional de Medicamentos Essenciais do Ministério da Saúde do Brasil. Cad Saude Publica 2014;30(9):1947-1956.

5. Kennedy-Dixon TG, Gossell-Williams M, Hall J, Anglin-Brown B. The prevalence of major potential drug-drug interactions at a University health centre pharmacy in Jamaica. Pharm Pract (Granada) 2015;13(4):601.

6. Tragni E, Casula M, Pieri V, Favato G, Marcobelli A, Trotta MG, Catapano AL. Prevalence of the prescription of potentially interacting drugs. PLoS One. 2013;8(10):e78827.

7. Brasil. Ministério da Saúde (MS). Portaria no 529 de $1^{\circ}$ de abril de 2013. Institui o Programa Nacional de Segurança do Paciente (PNSP) [portaria na Internet]. Diário Oficial da União; 2013. [acessado 2018 Jan 29]. Disponível em: http://bvsms.saude.gov.br/bvs/ saudelegis/gm/2013/prt0529_01_04_2013.html

8. Egger SS, Drewe J, Schlienger RG. Potential drugdrug interactions in the medication of medical patients at hospital discharge. Eur J Clin Pharmacol 2003;58(11):773-778.

9. Teka F, Teklay G, Ayalew E, Teshome T. Potential drug drug interactions among elderly patients admitted to medical ward of Ayder Referral Hospital, Northern Ethiopia: a cross sectional study. BMC Res Notes 2016;9(1):431. 
10. van Roon EN, van den Bemt PM, Jansen TL, Houtman NM, van de Laar MA, Brouwers JR. An evidence-based assessment of the clinical significance of drug-drug interactions between disease-modifying antirheumatic drugs and non-antirheumatic drugs according to rheumatologists and pharmacists. Clin Ther 2009;31(8):1737-1746.

11. Rosa MLG, Mesquita ET, Jorge AJL, Correia DMS, Lugon JR, Kang HC, Yokoo EM, Wahrlich V. Prevalence of chronic diseases in individuals assisted by the family health program in Niteroi, Brazil: evaluation of selection bias and protocol. Int J Med Res Health Sci 2015; 4(3):531-534.

12. Micromedex ${ }^{\circledR}$ Healthcare Series: MICROMEDEX 2.0 [plataforma na Internet]. [acessado 2018 Jan 29]. Disponível em: http://www.periodicos.capes.gov.br

13. Agência Nacional de Vigilância Sanitária (ANVISA). DCB - Denominações Comuns Brasileiras [página na Internet]. [acessado 2018 Jan 29]. Disponível em: http://portal.anvisa.gov.br/denominacao-comumbrasileira

14. Bleich GW, Bleich A, Chiamulera P, Sanches AC, Schneider DS, Teixeira JJ. Frequency of potential interactions between drugs in medical prescriptions in a city in southern Brazil. Sao Paulo Med J 2009;127(4):206-210.

15. Doubova Dubova SV, Reyes-Morales H, TorresArreola LP, Suárez-Ortega M. Potential drug-drug and drug-disease interactions in prescriptions for ambulatory patients over 50 years of age in family medicine clinics in Mexico City. BMC Health Serv Res 2007;7:147.

16. Codagnone-Neto V, Garcia VP, Helena ETS. Possible pharmacological interactions in hypertensive and/or diabetic elderly in family health units at Blumenau (SC). Braz J Pharm Sci 2010;46(4):795-804.

17. Venturini CD, Engroff P, Ely LS, Zago LF, Schroeter G, Gomes I, De Carli GA, Morrone FB. Gender differences, polypharmacy, and potential pharmacological interactions in the elderly. Clinics (Sao Paulo) 2011;66(11):1867-1872.

18. Leão DF, Moura CS, Medeiros DS. Avaliação de interações medicamentosas potenciais em prescrições da atenção primária de Vitória da Conquista (BA), Brasil. Ciên Saude Colet 2014;19(1):311-318.

19. Teixeira JJ, Crozatti MT, Santos CA, Romano-Lieber NS. Potential drug-drug interactions in prescriptions to patients over 45 years of age in primary care, southern Brazil. PLoS One 2012; 7(10):e47062.

20. Santos TRA, Silveira EA, Pereira LV, Provin MP, Lima DM, Amaral RG. Potential drug-drug interactions in older adults: A population-based study. Geriatr Gerontol Int 2017;17(12):2336-2346.
21. Sociedade Brasileira de Cardiologia. VI Diretrizes Brasileiras de Hipertensão. Rev Bras Hipertens 2010; 17(1):7-10.

22. U.S. Food \& Drug Administration (FDA). Drug Safety Communication: new restrictions, contraindications, and dose limitations for Zocor (simvastatin) to reduce the risk of muscle injury [página na Internet]. [acessado 2018 Jan 29]. Disponível em: https://www.fda.gov/ drugs/drug-safety-and-availability/fda-drug-safety-communication-new-restrictions-contraindications-and-dose-limitations-zocor

23. Sistema de Cadastramento e Acompanhamento de Hipertensos e Diabéticos (HIPERDIA) [página na Internet]. [acessado 2018 Jan 29]. Disponível em: http:// datasus.saude.gov.br/sistemas-e-aplicativos/epidemiologicos/hiperdia

24. Ministério da Saúde (MS). Farmácia Popular [página na Internet]. [acessado 2018 Jan 29]. Disponível em: http://www.saude.gov.br/acoes-e-programas/farmacia-popular

25. Patel PS, Rana DA, Suthar JV, Malhotra SD, Patel VJ. A study of potential adverse drug-drug interactions among prescribed drugs in medicine outpatient department of a tertiary care teaching hospital. J Basic Clin Pharm 2014;5(2):44-48.

26. Obreli Neto PR, Nobili A, Marusic S, Pilger D, Guidoni CM, Baldoni Ade O, Cruciol-Souza JM, Da Cruz AN, Gaeti WP, Cuman RK. Prevalence and predictors of potential drug-drug interactions in the elderly: a cross-sectional study in the brazilian primary public health system. J Pharm Pharm Sci 2012;15(2):344-354.

27. Dookeeram D, Bidaisee S, Paul JF, Nunes P, Robertson P, Maharaj VR, Sammy I. Polypharmacy and potential drug-drug interactions in emergency department patients in the Caribbean. Int J Clin Pharm 2017;39(5):1119-1127.

Artigo apresentado em 23/09/2017

Aprovado em 16/04/2018

Versão final apresentada em 18/04/2018 